\title{
THE DEVELOPMENT OF BIOLOGICAL PRODUCT FOR PLANT GROWING ON THE BASIS OF STREPTOMYCES ALBUS
}

\author{
Nataliia Levchyk \\ Laboratory of Biotechnology and Genetics, Department of Cultivated Flora \\ M. M. Gryshko National Botanical Garden of NAS of Ukraine \\ 1 Timiryazevska str., Kyiv, Ukraine, 01014
}

Alla Liubinska

Laboratory of Biotechnology and Genetics, Department of Cultivated Flora

M. M. Gryshko National Botanical Garden of NAS of Ukraine

1 Timiryazevska str., Kyiv, Ukraine, 01014

Tetiana Todosiichuk

Department of Industrial Biotechnology

National Technical University of Ukraine «Igor Sikorsky Kyiv Polytechnic Institute»

37 Peremohy ave., Kyiv, Ukraine, 03056

\author{
Jamal Rakhmetov \\ Department of Cultivated Flora \\ M. M. Gryshko National Botanical Garden of NAS of Ukraine \\ 1 Timiryazevska str., Kyiv, Ukraine, 01014 \\ Maria Diakova \\ Department of Industrial Biotechnology \\ National Technical University of Ukraine «Igor Sikorsky Kyiv Polytechnic Institute» \\ 37 Peremohy ave., Kyiv, Ukraine, 03056
}

\footnotetext{
Abstract

In this work the influence of experimental forms of biological product from Streptomyces albus UN44 on the growth processes of agricultural plants (wheat, peas, corn) was studied. The advantages of the preparation are demonstrated, which contains not only antibiotic and enzymatic complexes, synthesized by the producer, but also its biomass for the stimulation of seed germination processes, growth and development of the said plants seedlings.

The proposed process description of biological product Streptofungin-Phyto production in two forms is the basis for engineering development and operating regulations. The preparation can be developed as a suspension with a cell titer of $10^{9} \mathrm{CFU} / \mathrm{cm}^{3} \mathrm{in}$ PET-containers $(2-51)$ or as a dried mass in plastic bags $(1-2 \mathrm{~kg})$.

The biological product can be recommended for use on the stage of pre-planting treatment of seeds by soaking, as well as deep root watering and irrigation. The remedy is safe for humans, animals and plants and can be used together with other microbial mass-based biofertilizers.

Keywords: microbial biological product, finished dosage form, plant growing, cultivation, plant culture, process description.

\section{Introduction}

The biological activity of the Streptomyces albus culture (initially identified as recifensis) is primarily due to the production of hydrolytic enzymes complex, with which its ability to affect the growth processes of plants was also connected [1-3]. However, the recently discovered ability of selective strain of S. albus UN44 culture to exert antagonistic effect on fungi became the basis for considering the opportunities for production of different finished dosage forms of biological products for plant growing with combined effects - growth stimulation and protection against pathogens [4-6].
} 
The analysis of similar developments has shown that the finished dosage form of biological products, connected with their directions for use and storage life is especially important [7, 8]. Modern biological products (e. g. "Gaupsin”, Trichodermin BT - "Agrarian Biotechnology”, Ukraine) take into account the need to optimize the finished dosage form and determine the advantages of such forms as cell suspension, extracellular metabolite or dried cell biomass [9-11]. The most common form of biological products for plant growing is cell suspension, which sustainably simplifies the production technology due to the absence of biomass separation and purification stages, and thus minimization of material maintenance of production output $[12,13]$.

\section{Aim}

To study the influence of biological product samples based on S. albus UN44 on the growth processes of agricultural plants and develop the production flow chart for the chosen finished dosage form of the product.

\section{Materials and methods for determining the biological effect of experimental biological products on the basis of $S$. albus UN44 on the growth processes of agricultural plants}

\section{1. Microbial, plant cultures and equipment used for the study}

The culture of the producer of a bioactive substances complex Streptomyces albus UN44 and samples of biological products, based on it, from the collection of Department of Industrial Biotechnology of National Technical University of Ukraine "Igor Sikorsky Kyiv Polytechnic Institute" (Kyiv, Ukraine) were used in this study [5, 14]. Gauze's medium was used for the maintenance of the culture, and nutrient medium, based on soy-bean flour and starch - for the product biosynthesis $[5,15]$.

For determining the growth stimulating activity of the biological products the next plants were used: spring wheat Triticum L., garden pea Pisum sativum L., tyfon Brassica rapa L., maize Zea mays Bonduelle F1. from the collection of Department of New Cultures of M. M. Gryshko National Botanical Garden, NAS of Ukraine.

The experimental procedures and obtaining the experimental samples were carried out using next tools and equipment: rotary shaker (IKA HS/KS 260 basic, Germany), laboratory balance (OHAUS AR2140, China), pH meter (pH-150 MA, China), photocolorimeter (KFK-3-01, Russia), sterilizer (ГK-100-3M, Russia), fluorescent lamp (4000 1x).

\section{2. Methods of obtaining the biological products and determining their influence on} the growth processes of plants

Method of obtaining experimental samples of biological products. S. albus UN44 inoculum was grown in $250 \mathrm{~cm}^{3}$ flasks on the rotary shakers with a rotation speed of $200 \mathrm{rpm}$ and temperature $28 \pm 1{ }^{\circ} \mathrm{C}$ during $48 \mathrm{hrs}$. The flasks contained $50 \mathrm{~cm}^{3}$ of soy-bean flour and glucose based medium, in which $10 \%$ of cell suspension were added. Accumulation of the preparations was carried out in $750 \mathrm{~cm}^{3}$ flasks with $150 \mathrm{~cm}^{3}$ of soy-bean flour and starch based medium and $7 \%$ of inoculum during 50-55 hrs at a rotation speed of $240 \mathrm{rpm}$ and temperature $28 \pm 1{ }^{\circ} \mathrm{C}[15]$.

The concentration of viable cells in the culture (colony forming units, CFU) throughout the process of biosynthesis and in the final product was detected with microbiological method, which means planting the samples with corresponding dilution on Gauze's agar medium.

The experimental preparation of culture broth for plant growing was termed Streptofungin-Phyto-1 after a name of antifungal antibiotics complex synthesized by the producer - streptofungin [14]. The second rare form of the experimental preparation (Streptofungin-Phyto-2) was obtained after separating biomass from the culture broth with centrifugation and corresponded to the centrate, which contained metabolites of the culture. Experimental preparations were stored at $+4{ }^{\circ} \mathrm{C}$ and used for determination of stability during storage and studying the impact on the growth processes of plants.

Method of examination of growth stimulating effect of culture broth preparation. The study was carried out by the procedure of plant growing with the water-culture technique [16]. The right quantity of homogeneous seeds was soaked in weak potassium permanganate solution for $0,5 \mathrm{hrs}$. The seeds were germinated in Petri dishes on filter paper were moistened with distilled water 
at $24^{\circ} \mathrm{C}$ until chitting. The most viable seedlings were replanted into glass jars on filter paper, which was moistened, if necessary, with the solutions of experimental culture broth preparations with dilution of 1:20, 1:10 and with undiluted solution. Seedlings grown on the distilled water were taken as a reference sample. Cultivation was carried out at $25-26^{\circ} \mathrm{C}$ in conditions of artificial lighting, provided by 4000 lx fluorescent lamps [16]. Phenological observations of plants were carried out, and their external appearance was noted. The life conditions of seedling were evaluated on 3 , 4, 7, 10, 14 days by the morphometric parameters (height of the sprouts, root length, wet and dry weight of their elevated and underground parts). The experiments were repeated three times. The results of the study were processed statistically using Microsoft Office Excel 2007 programs.

\section{Results}

At the first stage of the study in above-mentioned conditions the accumulation of experimental biological products using the producer of complex of enzymes and antibiotics $S$. albus UN44 was conducted. Given that the products of biosynthesis are secreted by the culture into the environment, the next experimental samples were obtained: experimental sample of culture broth preparation (Streptofungin-Phyto-1) with the biomass of the producer, and the complex of all synthesized metabolites - centrate preparation (Streptofungin-Phyto-2) without biomass of the producer. During the study the impact of the experimental preparations on seed germination, growth and development on the virginal stage of ontogenesis of the selected plants was measured, and possible associated effects were tested.

The influence of Streptofungin-Phyto-2 preparation on germs of spring wheat Triticum L. revealed itself in significant inhibitory effect on the growth of wheat roots throughout the growing period (Table 1). At the end of the day 14 the length of the root in reference plants (not treated with the preparation) was $145,0 \pm 7,1 \mathrm{~mm}$, which is 1.5 times more than in treated plants $(101,0 \pm 5,0)$. The preparation had approximately the same effect on the development of the elevated parts of wheat - up to day 7 the length of stems of the treated sprouts also conceded to the reference plants and only later began to exceed it by $10-15 \%(223,7 \pm 10,8 \mathrm{~mm})$.

Table 1

The effects of treatment of spring wheat seedlings with Streptofungin-Phyto-2 preparation on the morphometric parameters of plants during their growing

\begin{tabular}{|c|c|c|c|c|c|}
\hline \multirow{3}{*}{ Treatment options } & \multicolumn{5}{|c|}{ Growing duration, days } \\
\hline & 3 & 4 & 7 & 10 & 14 \\
\hline & \multicolumn{5}{|c|}{ Root length, mm } \\
\hline $\begin{array}{l}\text { preparation dilution } \\
\qquad(1: 20)\end{array}$ & $23,0 \pm 1,1$ & $35,9 \pm 1,7$ & $89,3 \pm 2,2$ & $93,3 \pm 4,6$ & $101,0 \pm 5,0$ \\
\hline $\begin{array}{c}\text { no treatment } \\
\text { (reference sample) }\end{array}$ & $44,3 \pm 2,1$ & $64,4 \pm 3,2$ & $117,00 \pm 5,7$ & $131,7 \pm 6,5$ & $145,0 \pm 7,1$ \\
\hline \multicolumn{6}{|c|}{ Stem length, mm } \\
\hline $\begin{array}{l}\text { preparation dilution } \\
\qquad(1: 20)\end{array}$ & $14,7 \pm 0,7$ & $33,7 \pm 1,5$ & $138,8 \pm 6,6$ & $191,7 \pm 8,5$ & $223,7 \pm 10,8$ \\
\hline $\begin{array}{c}\text { no treatment } \\
\text { (reference sample) }\end{array}$ & $22,2 \pm 1,0$ & $40,2 \pm 2,0$ & $138,7 \pm 6,4$ & $176,7 \pm 7,9$ & $201,7 \pm 9,1$ \\
\hline
\end{tabular}

The effect of another preparation - Streptofungin-Phyto-1 was first estimated by the effect on the germination of seeds in samples of spring wheat and peas. Six-day seedlings of these plants, treated with 1:20 dilution of the preparation, exceeded the reference plants sufficiently by the intensity of germination (Fig. 1). Thus, the intensity of wheat germination after the preparation treatment of seeds increased almost in two times, and the germination of peas - in 3-4 times. 


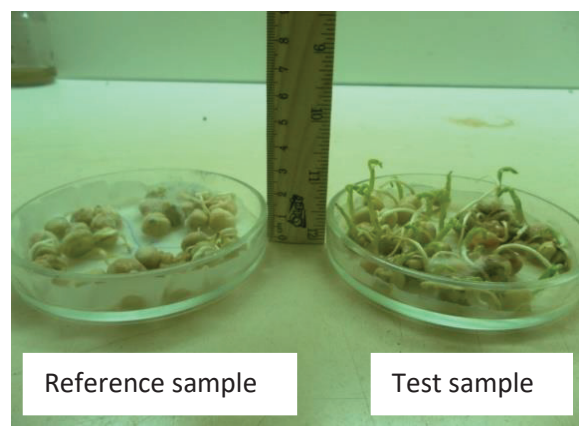

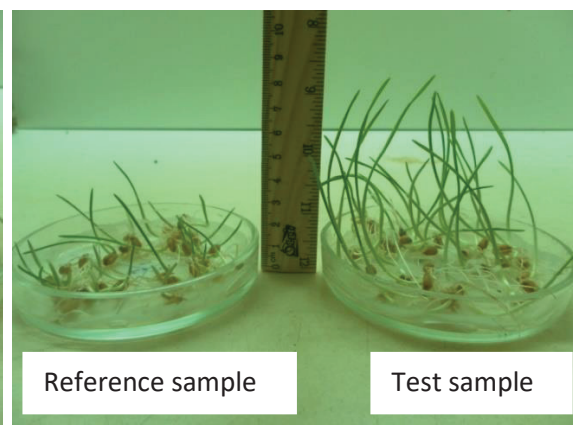

$b$

Fig. 1. The effects of treatment with Streptofungin-Phyto-1 preparation (dilution 1:20) on seed germination of: $a$ - peas; $b$ - spring wheat

During a series of these experiments the effect of the Streptofungin-Phyto-1 preparation on the mass of plants was also determined. The difference in preparation effects on increasing the mass of peas and wheat - both elevated and underground parts - was noted (Table 2).

Table 2

The mass of seedlings of peas and wheat, treated with the Streptofungin-Phyto-1 preparation

\begin{tabular}{|c|c|c|c|c|}
\hline \multirow{3}{*}{ Treatment options } & \multicolumn{4}{|c|}{ Seedlings mass, $\mathrm{g}$} \\
\hline & \multicolumn{2}{|r|}{ Wet weight } & \multicolumn{2}{|c|}{ Dry weight } \\
\hline & Root & Stem & Root & Stem \\
\hline \multicolumn{5}{|c|}{ Peas Pisum sativum L. } \\
\hline preparation dilution (1:10) & $2,00 \pm 0,05$ & $2,07 \pm 0,06$ & $0,14 \pm 0,01$ & $0,16 \pm 0,01$ \\
\hline $\begin{array}{c}\text { no treatment } \\
\text { (reference sample) }\end{array}$ & $1,02 \pm 0,01$ & $0,98 \pm 0,02$ & $0,08 \pm 0,02$ & $0,10 \pm 0,01$ \\
\hline \multicolumn{5}{|c|}{ Spring wheat Triticum $L$. } \\
\hline preparation dilution (1:10) & $0,66 \pm 0,05$ & $0,90 \pm 0,03$ & $0,14 \pm 0,01$ & $0,11 \pm 0,01$ \\
\hline $\begin{array}{c}\text { no treatment } \\
\text { (reference sample) }\end{array}$ & $0,81 \pm 0,07$ & $1,10 \pm 0,08$ & $0,12 \pm 0,01$ & $0,11 \pm 0,01$ \\
\hline
\end{tabular}

At the final stage of this series of experiments the effects of the preparation on growth processes of peas in soil were determined. The determined concentration and schemes of treatment (preplant, periodic irrigation) were used in this experiment to determine the effect of preparation on growth of tyfon Brassica rapa L. and corn Zea mays Bonduelle F1 in soil (Table 3).

In accordance with the previously described results, seeds were soaked in water to swell before seeding and thereafter every 2 days irrigated with the preparation in a dilution 1:20.

Taking these facts into account, culture broth suspension and dry preparation of the culture broth were proposed as the final forms of the product. The identified concentration of cells at the end of cultivation process of the producer was $10^{8}-10^{9} \mathrm{CFU} / \mathrm{cm}^{3}$, corresponding to the average concentration of cells in the similar developments, mentioned above. Therefore, it is inappropriate to additionally concentrate the culture broth during the production of the preparation. The other reason for this is the shown growth stimulating activity of the preparation with a dilution of 10-20 times.

The developed variations of the process description of obtaining the preparations for plant growing show the sequence of stages and production conditions for liquid and dry formulations of Streptofungin-Phyto preparation (Fig. 2). 
Table 3

Morphometric parameters of plants influenced by the Streptofungin-Phyto-1 preparation during germination in soil

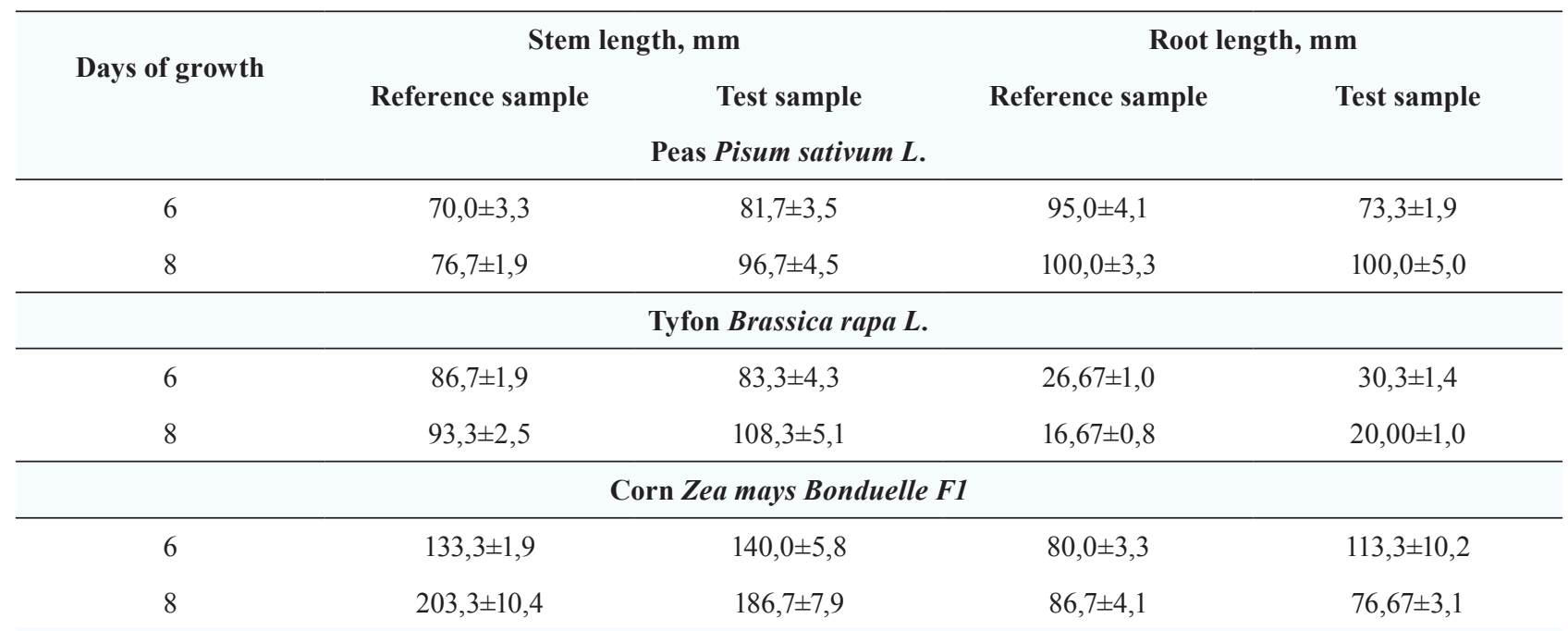

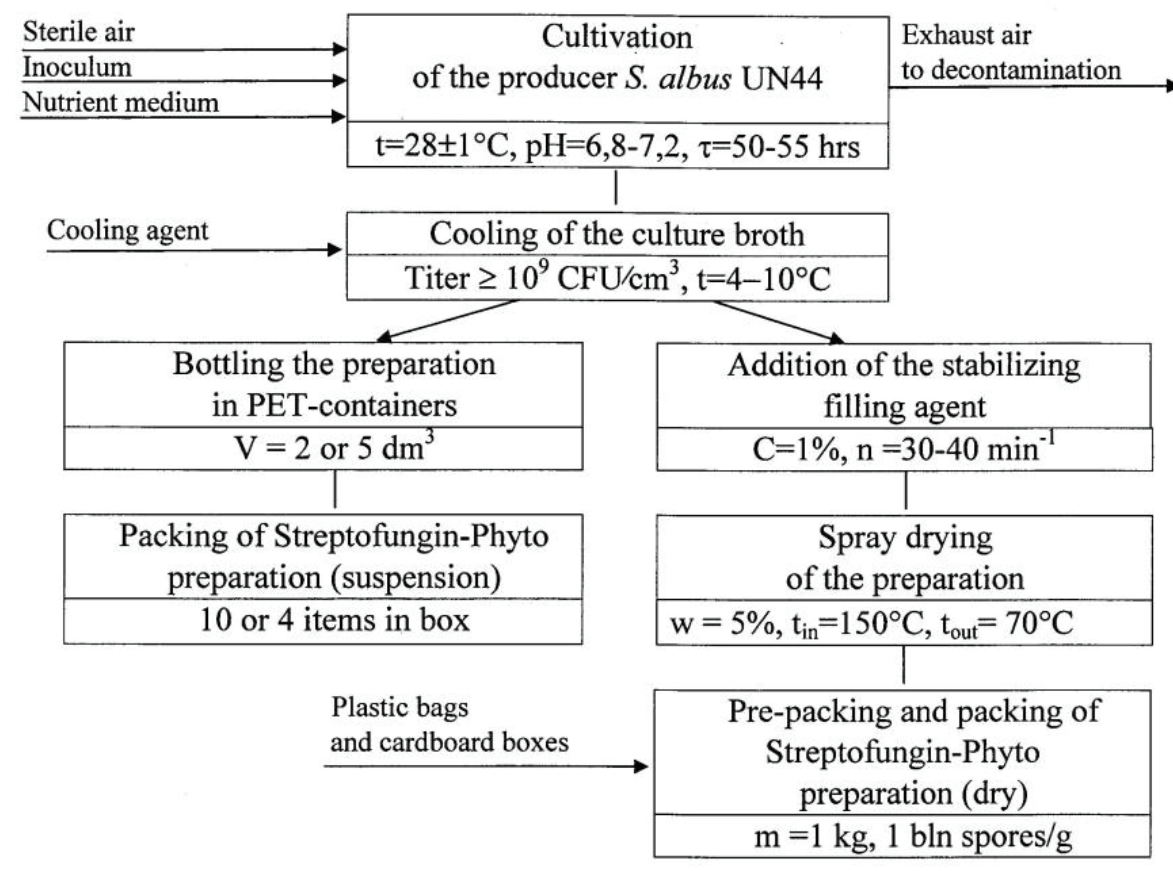

Fig. 2. Process description of obtaining the biological product Streptofungin-Phyto in the form of suspension and in dried formulation

The studies of stability of S. albus UN 44 culture broth during 3 months storage showed that the concentration of vegetative cells of the producer decreased by $5-10 \%$ at the temperature of $4-10{ }^{\circ} \mathrm{C}$, and the concentration of spores - within the margin of error. Therefore, when obtaining the liquid preparation, it is possible not to add the stabilizer and store the product at the temperature of $4-10{ }^{\circ} \mathrm{C}$ for $2-3$ months.

However, given the results on the positive impact of $\mathrm{SiO}_{2}$ on the stability of enzymes of the culture (while developing the immobilized enzyme form) and also literature data [15, 17], it was proposed to use it at a concentration of $1 \%$ as a stabilizer for a dry formulation. The concentration of spores of the culture in the finished dried preparation was about $1 \mathrm{bln}$ spores $/ \mathrm{g}$. Packaged in plastic bags of $1-2 \mathrm{~kg}$, the preparation can be stored up to 2 years at temperatures up to $20^{\circ} \mathrm{C}$ and 
diluted with water or nutritional composition before using for a fast recovery of culture's activity, as the similar microbial preparation $[5,7,10]$.

\section{Discussion of the results}

It is clearly inappropriate to use the preparation centrate Streptofungin-Phyto-2 (Table 1) to stimulate the growth of wheat at the beginning of its cultivation. A slight stimulation of stem growth after 10-14 days in soil conditions may be ineffective, but could be the basis for developing individual schemes of use for such product at the later stages of growth.

The difference in effects of Streptofungin-Phyto-1 preparation treatment on germinating capacity of plants was also noted (Fig. 1). The germinating capacity of wheat in the reference sample was $100 \%$, and did not differ statistically in the test sample, reaching $97 \%$. However, only $77 \%$ of seeds germinated in the reference batch of peas, and in the test samples the germinating capacity increased to $87 \%$. The determined stimulating effect of the preparation on the germinating capacity of plants, apparently, has species-specific dependence and requires more detailed spectrum of such cultures and patterns of use. Expanding research field in this direction is reasonable in case of using the built-up experimental batch of the preparation following the proposed technology.

The treatment of wheat seedlings with the experimental preparation Streptofungin-Phyto-1 (dilution 1:10) during cultivation does not lead to any significant effects on the accumulation of mass by the plants, decreasing the most by $20 \%$ in the values of wet weight in reference samples, and virtually identical in the values of dry weight (Table 2). Another effect has the preparation on the peas seedlings: treated plants dominated reference samples by $96-111 \%$ in wet weight and by $75-78 \%$ in dry weight. It is obvious that preparation has a significant growth stimulating effect exactly on the peas plant, which is proved not only by the rates of stimulation of seed germination (Fig. 1), but also the presented rates of mass accumulation of the plants.

After analyzing the data of the experiment (Table 3) it can be concluded, that the preparation affects the plants in both laboratory and soil conditions, stimulating one cultures and inhibiting others at different periods of their development. For example, stem growth is observed to be stimulated in pea seedlings, but during the first week of exposure - a significant inhibition of root growth. The effect on tyfon is characterized with the stimulation of both root and stem growth.

The results of the study indicate the ability of the studied preparation to influence the growth processes of crops, stimulate them, but directions for the use of the product need further research and determination for the certain species of plants. It is also obviously reasonable to use the product, which contains not only metabolites of the producer, but also its cells and biomass (Table 1, 2), that after reaching the soil or the plant propagate themselves and continue to secrete biologically active products into environment. The same techniques are used in the development of numerous microbial preparations for agriculture (including plant growing) [18-20].

As the culture of $S$. albus was isolated from soil, where much of the microbial association is represented by actinomycetes in particular, clear is its safety for the environment, which is approved by the statement of nonpathogenicity.

\section{Conclusion}

Thus, following the results of the conducted studies it was established that the biological product Streptofungin-Phyto of culture broth of Str. albus UN 44 has effect on agricultural plants in the laboratory and in soil conditions, which is reflected in the growth rate of the elevated and underground parts of plants and increase in their dry and wet weight. The effect of the product can be stimulating or inhibitory and depends on the plant species, stage of its ontogeny and concentration (dilution) of the preparation. Inhibitory effect of the product at certain stages of ontogeny of plants can be followed with the stimulation of growth processes and vice versa. Overall performance of experimental preparation is characterized by the inhibition of root growth and stimulation of stem growth in the studied plants. 
It was determined, that the best effects on agricultural plants in laboratory conditions have the preparation with the dilution 1:10, under influence of which growth processes stimulation and weight increase take place. These processes are a sign of the intensification of photosynthesis and metabolism in the tested plants.

The obtained results are the reference for the optimization of the methods and modes of application of the biological product Streptofungin-Phyto for certain agricultural plants. The proposed process description for obtaining the product is the basis for further development of product technology and standard documentation.

\section{References}

[1] Babenko, L. P., Sokolova, I. E. (2009). Diosynthetical activity of Streptomyces recifensis transformants. Visnyk of Dnipropetrovsk University. Biology, ecology, 17 (1), 10-15. doi: 10.15421/010902

[2] Zhernosekova, I. V. (2012). Streptomycetes biosynthesis processes in the presence of vegetable oils. Visnyk of Dnipropetrovsk University. Biology, ecology, 20 (2), 25-29. doi: 10.15421/011224

[3] Alekseenko, O., Gernosekova, I., Vinnikov, A. (2011). Investigation of Streptomyces recifensis var. lyticus exometabolytes influences on the Pleurotus ostreatus fungi growth. Microbiol. and biotechnol. J., 2 (14), 41-47.

[4] Todosiichuk, T., Sichel, L. (2015). A novel producer of the antibiotic substances Streptomyces albus UN 44. Materials of 14th International Conference on the Chemistry of Antibiotics and Other Bioactive Compounds. Galveston, TX, USA, 22.

[5] Todosiichuk, T., Pokas, O., Yaremchuk, S. (2015). Streptomyces albus strain - producer of a complex of antibiotic substances and a complex of antibiotic substances with antifungal activity. Patent for an invention C12N1/20, 109568 UA, IPC (2015). № a 2013 11432, declareted: 27.09.2013; published: 10.09.2015. Byul. № 17.

[6] Halatyan, R., Jevtushenko, I., Sokolova, I. (2011). Production of the avermectyns by the Streptomyces recifensis var. lyticus $2 \mathrm{P}-15$ strain. Materials of 5 th International Conference «Chemistry and modern technologies». Dnipropetrovsk, 511.

[7] Babalola, O. O. (2010). Beneficial bacteria of agricultural importance. Biotechnology Letters, 32 (11), 1559-1570. doi: 10.1007/s10529-010-0347-0

[8] Rabinovich, G. Y., Smirnova, Y. D., Kovalev, N. G. (2016). Testing of two types of LPB as soil fertilizer biopreparation in the model experiment. Russian Agricultural Sciences, 42 (1), 58-61. doi: 10.3103/ s1068367416010171

[9] Maxymov, I., Mangesov, V., Kurchaev, E. (2010). Influence of the Bynoram fungicide on the safety of food root crops of carrots. Young scientist, 11 (22), 207-209.

[10] Morgun, V., Kots, S., Kirichenko, E. (2009). Growth stimulating rhizobacteria and their practical application. Physiology and Biochenistry of Agricultural Plants, 41 (3), 187-207.

[11] Shternshis, M. (2012). Tendencies of development of microbial products biotechnology for plant protection in Russia. Tomsk State University Bulletin. Biology, 18 (2), 92-100.

[12] Hamedi, J., Mohammadipanah, F. (2014). Biotechnological application and taxonomical distribution of plant growth promoting actinobacteria. Journal of Industrial Microbiology \& Biotechnology, 42 (2), 157-171. doi: 10.1007/s10295-014-1537-x

[13] Zavalyna, A., Kogemyakova, A. (2010). New technologies of the production and application of the preparations with the complex action. SP: Chemprint, 4, 12-18.

[14] Todosiichuk, T., Pokas, O., Yaremchuk, S. (2014). Patent for an invention 88598 UA, IPC (2014) C12N1/00. Streptofungin with antifungal activity. № u 2013 11433, declareted: 27.09.2013; published: 25.03.2014. Byul. № 6 .

[15] Grygoryeva, M. (2009). Biotechnology of immobilized hydrolytic enzyme product Cytorecifen-M. Kyiv, 157.

[16] Voitsehivska, O., Kapustyan, A., Kosyk, O. et. al.; Parshikova, T. V. (Ed.) (2010). Physiology of plants. Lutsk: Teren, 420. 
[17] Guisan, J. M. (Ed.) (2006). Immobilization of Enzymes and Cells. Methods in Biotechnology, 128. doi: 10.1007/978-1-59745-053-9

[18] Petrova, S., Parahin, N. (2013). Microbial preparations as a mean of forming effective plantmicrobial systems. Leguminous and groat cultures, 2 (6), 86-91.

[19] Sadykova, V. S., Kurakov, A. V. (2013). Prospects for the use of strains of the genus Trichoderma to obtain vermicomposts with fungicides and growth-stimulating properties. Russian Agricultural Sciences, 39 (3), 257-260. doi: 10.3103/s106836741303018x

[20] Romanenko, N., Popov, I., Tabolin, S., Bugaeva, E. N, Zaec, V. G. (2008). Perspectives of using antagonist bacteria against the most phytopathogenic species of nematodes, viruses and fungi. Agro XXI, $1-3,23-27$.

\title{
THE STUDY OF FATTY AND ORGANIC ACIDS COMPOSITION IN QUINCE LEAVES AND FRUITS (CYDONIA OBLONGA MILL)
}

\author{
Tetiana Dzhan \\ Department of Microbiology, Modern Biotechnology, \\ Ecology and Immunology \\ Open International University of Human Development "Ukraine" \\ 23 Lvivska str., Kyiv, Ukraine, 03179 \\ zakucilo@gmail.com
}

\begin{abstract}
The composition of fatty and organic acids in quince leaves and fruits (Cydonia oblonga Mill.) of selection of M. M. Grishko National Botanical Garden of National Academy of Sciences of Ukraine sorts was determined by gas chromatography-mass spectrometry method. Six sorts of quince were examined: "Academichna", "Kashchenka N18", "Studenrka', "Oranzheva", "Maria", "Darunok onuku". The presence of 36 fatty acids and 32 organic acids in leaves and fruits of the quince were identified and determined their content. Therefore, main acids are oxalic, citric, palmitic and linolenic acids in quince leaves and malic, palmitic and linoleic acids in quince fruits. The highest content of all acids were identified in the quince leaves of sort "Maria". Moreover, a quite high content of succinic acid in the quince leaves was detected.
\end{abstract}

Keywords: quince, fatty acids, organic acids, gas chromatography-mass spectrometry method.

\section{Introduction}

The quince (Cydonia oblonga Mill.) is recognized as a raw material for gelling products, due to the high content of pectin substances which are natural sorbents and they main function is removing body toxins and radio nuclides. The quince has known from ancient times, but unfortunately, is not always sufficiently evaluated. Now quince plays an important role in the economy of some countries and in other countries it is used only to meet domestic needs. Natural habitat of quince is Eastern and Southern Transcaucasia, Asia Minor, Iran. In other parts of the world the wild quince has not been discovered. The quince is cultivated in more than 40 countries, mainly in the humid continental and subtropical climates. But mainly in many countries its plantations are small and the quince may only be found in private gardens.

Study of the chemical composition and pharmacological activity of quince has shown positive prospects of quince fruits and leaves usage for drugs creation [1-15].

In Ukraine, quince is grown in small amounts; mainly in the south-western and southern regions with an average temperature from $10^{\circ} \mathrm{C}$ to $15^{\circ} \mathrm{C}$ and the length of growing season 